\title{
Use of Concomitant Antibiotics During Treatment for Clostridium difficile Infection (CDI) in Pediatric Inpatients: An Observational Cohort Study
}

\author{
Vanessa W. Stevens - Cary Thurm - Elyse M. Schwab • Matthew P. Kronman • \\ Jeffrey S. Gerber · Samir S. Shah · Jason G. Newland · Joshua Courter • \\ Sarah Parker · Thomas V. Brogan · Adam L. Hersh
}

Received: January 27, 2016/Published online: March 14, 2016

(C) The Author(s) 2016. This article is published with open access at Springerlink.com

\begin{abstract}
Concomitant antibiotic use during treatment for Clostridium difficile infection (CDI) increases the risk of recurrence. Across a network of children's hospitals, $46 \%$ of patients treated for CDI received concomitant antibiotics for a median of 7 days. Concomitant antibiotic use was more common among patients with malignancies, and solid organ or bone marrow
\end{abstract}

Enhanced content To view enhanced content for this article go to http://www.medengine.com/Redeem/ 5A44F0605DBC5E45.

V. W. Stevens $(\varangle)$ - E. M. Schwab

Department of Pharmacotherapy, University of Utah College of Pharmacy, 30 South 2000 East, Rm 4961, Salt Lake City, UT 84121, USA

e-mail: vanessa.stevens@pharm.utah.edu

C. Thurm

Children's Hospital Association, Washington D.C., MD, USA

M. P. Kronman · T. V. Brogan

Department of Pediatrics, Seattle Children's

Hospital, University of Washington, Seattle, WA, USA

\section{J. S. Gerber}

Division of Infectious Diseases,

Children's Hospital of Philadelphia,

Philadelphia, PA, USA transplant. Unnecessary concomitant antibiotic use in CDI patients is a potential target for pediatric antimicrobial stewardship.

Keywords: Clostridium difficile infection; Concomitant antibiotics; Pediatric

\section{INTRODUCTION}

Clostridium difficile infection (CDI), a common hospital-acquired infection, is associated with $10-25 \%$ of all cases of antibiotic-associated diarrhea [1]. Treatment of pediatric CDI is

\section{S. S. Shah $\cdot$ J. Courter}

Division of Hospital Medicine, Cincinnati

Children's Hospital Medical Center, Cincinnati,

$\mathrm{OH}, \mathrm{USA}$

J. G. Newland

Division of Infectious Diseases, Children's Mercy Hospitals and Clinics, University of Missouri, Kansas City, MO, USA

\section{S. Parker}

Department of Pediatrics, University of Colorado School of Medicine and Children's Hospital Colorado, Aurora, CO, USA

\section{A. L. Hersh}

Division of Infectious Diseases, Department of Pediatrics, University of Utah School of Medicine, Salt Lake City, UT, USA 
based largely on recommendations for the treatment of adults [2] and discontinuation of the inciting antibiotic is recommended when feasible [3]. Concomitant antibiotic use targeting other infections during treatment for CDI has been associated with a four- to five-fold increase in the risk of CDI recurrence, and should be avoided when possible [4-6]. Nearly $60 \%$ of adult patients being treated for CDI receive concomitant antibiotics [7], but the extent to which concomitant antibiotics are prescribed during treatment for pediatric CDI is unknown. We hypothesized that rates of concomitant antibiotic use among pediatric CDI patients would be similar to those observed among adults.

\section{METHODS}

\section{Study Design and Patient Selection}

The Pediatric Health Information System (PHIS) is a database containing administrative data on billed services (including pharmacy, radiology, laboratory and other services) from 43 freestanding children's hospitals participating in the Children's Hospital Alliance (CHA). Data from the participating centers are compiled, de-identified, and validated before being released for use by researchers. We conducted a retrospective multi-center cohort study of pediatric hospitalizations using the PHIS database. Patients were eligible for inclusion if they were admitted to a PHIS hospital between July 1, 2008 and December 31, 2013 and were greater than 6 months but less than 18 years at admission. A case of CDI required both an International Classification of Diseases, Ninth Revision, Clinical Modification (ICD9-CM) code for CDI (008.45), and the receipt of CDI antibiotics, defined as either oral or intravenous metronidazole or oral vancomycin. Case-finding approaches using administrative data have been shown to have greater than 99\% specificity among both children [8] and adults [9]. This study has been reviewed and deemed exempt by the Institutional Review Board (IRB) of the University of Utah.

\section{Demographic and Clinical Characteristics}

Patient demographic and clinical information was collected, including age at the time of hospitalization with CDI, race, gender, presence of comorbidities based on complex chronic condition [10], and initial CDI episode classification as presumed hospital-onset or community-onset based on the timing of anti-CDI treatment initiation [11]. Patients who initiated CDI treatment within 2 days of hospital admission were classified as community-onset, and patients who initiated CDI treatment 3 or more days following admission were classified as hospital-onset. A subgroup of immunocompromised patients including those with malignancy, solid organ, or bone marrow transplant was identified based on administrative codes. A diagnosis of cancer was based on Feudtner's complex chronic condition classification [10]. Solid organ transplant (SOT) was defined using ICD9-CM codes for lung and heart transplant $(33.5 \times$, 33.6, 37.51), liver transplant $(50.5 \times)$, and kidney transplant $(55.6 \times)$. Bone marrow transplant (BMT) was defined using ICD9-CM codes for allogeneic and autologous transplant (41.00-41.03, 41.09).

\section{Concomitant Antibiotics}

Concomitant antibiotic exposure was defined as receipt of systemic antibiotics other than CDI antibiotics) for at least three consecutive days 
during the treatment course for CDI. This definition was chosen in order to allow for some delay in the time required to discontinue antibiotics following a CDI diagnosis. Concomitant antibiotics were categorized according to major classes, including anti-pseudomonal beta-lactams (APBL), cephalosporins, aminoglycosides, fluoroquinolones, macrolides, penicillins, and others. Antibiotics commonly used for prophylaxis and other non-infectious indications were excluded because their ongoing use may not have been modifiable despite an active CDI. The excluded agents were: intramuscular bacitracin, oral colistimethate, erythromycin, gentamicin, neomycin, paromomycin, rifaximin, sulfamethoxazole-trimethoprim, sulfasalazine, and tobramycin. The number of concomitant antibiotics received and duration of concomitant therapy were also recorded.

\section{Data Analysis}

Standard descriptive statistics (e.g., frequencies, percentages, medians) were used to characterize concomitant antibiotic use by patient subgroup and describe frequency and duration of use. All statistical analyses were conducted using SAS v.9.2 (Cary, NC, USA).

\section{RESULTS}

Of 16,777 children with CDI during the study period, 7638 (45.5\%) received concomitant antibiotics for $\geq 3$ days during treatment for CDI (Table 1). This proportion has decreased over time, from $49.7 \%$ in 2008 to $41.9 \%$ in 2013. There was significant variability in the proportion of patients receiving concomitant antibiotic therapy across hospitals, ranging from $18.1 \%$ to $58.0 \%$. More than $85 \%$ of patients received metronidazole monotherapy for the treatment of CDI. Concomitant antibiotic use was less common among patients with community-onset CDI (37.7\%), without comorbid conditions (24.0\%), and those receiving oral vancomycin alone for CDI treatment $(37.1 \%)$. Patients with malignancy $(64.4 \%)$, solid organ transplant $(67.2 \%)$, and BMT (86.9\%) had the highest rates of concomitant antibiotic use. Younger patients (6-12 months) had a slightly lower rate of concomitant antibiotic use (40.7\%). Overall, patients with concomitant antibiotic use received a median of 2.0 (IQR 1.0) non-CDI antibiotics during CDI treatment for a median of 7.0 (IQR 7.0) days. The most commonly received concomitant antibiotic class was anti-pseudomonal beta-lactams (95\%), either as a single agent or in combination with other agents. Other commonly received single-agent concomitant antibiotics included: cephalosporins, penicillins, and vancomycin. More than $50 \%$ of patients with any concomitant antibiotics received an anti-pseudomonal beta-lactam in combination with intravenous vancomycin.

\section{DISCUSSION}

Across 43 children's hospitals, nearly half of patients treated for CDI received at least 3 days of concomitant antibiotics. The proportion on patients receiving concomitant antibiotic therapy during CDI treatment varied significantly across hospitals, and appears to be decreasing slightly over time. Concomitant antibiotic use was most common among patients with immune compromising conditions including malignancy and solid organ or bone marrow transplant. 
Table 1 Concomitant antibiotic receipt by patient subgroup

\begin{tabular}{|c|c|c|}
\hline Characteristics & Total $n$ & $n$ (\%) with concomitant antibiotics \\
\hline Overall & 16,777 & $7638(45.5)$ \\
\hline \multicolumn{3}{|l|}{ Age, years } \\
\hline 6 months to $>1$ year & 1448 & $590(40.7)$ \\
\hline $1-4$ years & 6350 & $2955(46.5)$ \\
\hline $5-12$ years & 4399 & $2028(46.1)$ \\
\hline $13-18$ years & 4580 & $2065(45.1)$ \\
\hline Sex, male & 8924 & $4117(46.1)$ \\
\hline Sex, female & 7853 & $3521(44.8)$ \\
\hline \multicolumn{3}{|l|}{ Race } \\
\hline Non-hispanic white & 9284 & $3968(42.7)$ \\
\hline Non-hispanic black & 2118 & $994(46.9)$ \\
\hline Hispanic & 3220 & $1631(50.7)$ \\
\hline Asian & 467 & $231(49.5)$ \\
\hline Other & 1688 & $814(48.2)$ \\
\hline \multicolumn{3}{|l|}{ CDI onset } \\
\hline Community & 10,695 & $4030(37.7)$ \\
\hline Hospital & 6082 & $3608(59.3)$ \\
\hline \multicolumn{3}{|l|}{ No. of comorbidities } \\
\hline 0 & 4215 & $1010(24.0)$ \\
\hline 1 & 7762 & $3751(48.3)$ \\
\hline$>1$ & 4800 & $2877(59.9)$ \\
\hline \multicolumn{3}{|l|}{ Immunocompromised } \\
\hline Malignancy & 5257 & $3387(64.4)$ \\
\hline Solid organ transplant & 189 & $127(67.2)$ \\
\hline Bone marrow transplant & 221 & $192(86.9)$ \\
\hline Any immune compromise & 5484 & $3547(64.7)$ \\
\hline \multicolumn{3}{|l|}{ Initial treatment regimen } \\
\hline Metronidazole & 14,354 & $6663(46.4)$ \\
\hline Vancomycin & 1448 & $537(37.1)$ \\
\hline Combination & 975 & $438(44.9)$ \\
\hline
\end{tabular}

Excluded antibiotics: intramuscular bacitracin; oral colistimethate, erythromycin, gentamicin, neomycin, paromomycin, rifaximin, sulfamethoxazole-trimethoprim, sulfasalazine, and tobramycin. Data are no. (\%), unless otherwise indicated $I Q R$ interquartile range, CDI Clostridium difficile infection, SOT solid organ transplant, BMT bone marrow transplant 
Anti-pseudomonal beta-lactam (APBL) antibiotics were the most frequently prescribed class and the median duration of therapy was 7 days. Our findings suggest that concomitant antibiotic use is widespread in children's hospitals during CDI therapy. Concomitant therapy may contribute to recurrent infection among pediatric patients [12], and represents an important stewardship target within the context of larger efforts to reduce the spread and impact of $C$. difficile in children's hospitals.

The high rates of concomitant antibiotics received by patients during CDI treatment have significant clinical implications, particularly among patients who are already at increased risk of recurrent CDI secondary to underlying comorbidity. The first recommendation for treatment of CDI is to discontinue the use of inciting or unnecessary antibiotics [3]. The increase in risk of recurrent CDI while patients are on antibiotics is due to the continued disruption of the normal gut microbiota. Since C. difficile is ubiquitous and common in the healthcare environment, patients will remain at risk of CDI while the normal flora are suppressed. Thus, non-CDI-targeted antibiotics should not be used in the peri-infection period unless absolutely necessary.

Antibiotics are commonly overused, and are a major contributor to the increasing rates of drug-resistant infections [13]. Evidence suggests that $25 \%$ or more of antibiotic prescriptions across inpatient and outpatient settings are not indicated [14, 15]. However, concomitant therapy may not always be avoidable in patients with CDI. For example, management of patients with febrile neutropenia requires the initiation of empiric antibiotics to avoid severe infections [16]. Not all antibiotics may confer an increased risk of CDI or recurrent CDI [17]. Future work in this area could help clarify which agents are most appropriate for patients in whom concomitant antibiotic use is unavoidable. Antimicrobial stewardship efforts in these patients could instead focus on appropriate antibiotic selection, duration optimization, and management of redundant coverage.

This study is subject to a number of limitations, which should be considered when interpreting our results. First, we used ICD-9 codes together with CDI treatment rather than clinical symptoms to identify patients with CDI. It is well known that CDI diagnosis in children is problematic [18]. We excluded patients less than 6 months of age in order to eliminate those patients least likely to have a clinically meaningful CDI. Diagnosis among patients $>6$ months to 2 years of age remains an area of some uncertainty. However, our results indicate that patients in this age group are being diagnosed with and treated for CDI, which may represent an area for further stewardship activities. Second, the PHIS database only contains administrative data on inpatient admissions. Since the majority of patients develop recurrent CDI in the outpatient setting, we were unable to follow patients for recurrence. Future work should focus on exploring the relationship between concomitant antibiotics and the risk of recurrent infection.

\section{CONCLUSIONS}

We found that concomitant antibiotic exposure during CDI treatment is common for hospitalized children, especially among those with immunocompromising conditions. Concomitant therapy was frequently prescribed for prolonged durations. Antimicrobial stewardship programs should consider interventions to identify potentially modifiable or unnecessary concomitant 
therapy, especially for immunocompromised patients and others at a higher risk of recurrent CDI.

\section{ACKNOWLEDGMENTS}

No funding or sponsorship was received for this study or publication of this article. All named authors meet the International Committee of Medical Journal Editors (ICMJE) criteria for authorship for this manuscript, take responsibility for the integrity of the work as a whole, and have given final approval to the version to be published. Selected results from this study were presented in poster format at the ID Week in Philadelphia, PA on October 8, 2014.

Disclosures. Vanessa W. Stevens, Cary Thurm, Elyse M. Schwab, Matthew P. Kronman, Jeffrey S. Gerber, Samir S. Shah, Jason G. Newland, Joshua Courter, Sarah Parker, Thomas V. Brogan, and Adam L. Hersh declare that they have no conflicts of interest relevant to this article.

Compliance with Ethics Guidelines. This study was reviewed and deemed exempt by the IRB of the University of Utah.

Open Access. This article is distributed under the terms of the Creative Commons Attribution-NonCommercial 4.0 International License (http://creativecommons.org/licenses/ by-nc/4.0/), which permits any noncommercial use, distribution, and reproduction in any medium, provided you give appropriate credit to the original author(s) and the source, provide a link to the Creative Commons license, and indicate if changes were made.

\section{REFERENCES}

1. Bartlett JG. Clostridium difficile: history of its role as an enteric pathogen and the current state of knowledge about the organism. Clin Infect Dis. 1994;18(Suppl 4):S265-72.

2. Schutze G, Willoughby R. Clostridium difficile infection in infants and children. Pediatrics. 2013;131(1):196-200.

3. Cohen SH, Gerding DN, Johnson S, et al. Clinical practice guidelines for Clostridium difficile infection in adults: 2010 update by the society for healthcare epidemiology of America (SHEA) and the infectious diseases society of America (IDSA). Infect Control Hosp Epidemiol. 2010;31(5):431-55.

4. Shivashankar R, Khanna S, Kammer PP, et al. Clinical predictors of recurrent Clostridium difficile infection in out-patients. Aliment Pharmacol Ther. 2014;40(5):518-22.

5. Tschudin-Sutter S, Tamma PD, Milstone AM, Perl TM. The Prediction of complicated Clostridium difficile infections in children. Infect Control Hosp Epidemiol. 2014;35(7):901-3.

6. Deshpande A, Pasupuleti V, Thota P, et al. Risk factors for recurrent Clostridium difficile infection: a systematic review and meta-analysis. Infect Control Hosp Epidemiol. 2015;36(4):452-60.

7. Harpe SE, Inocencio TJ, Pakyz AL, Oinonen MJ, Polk RE. Characterization of continued antibacterial therapy after diagnosis of hospital-onset Clostridium difficile infection: implications for antimicrobial stewardship. Pharmacotherapy. 2012;32(8):744-54.

8. Shaklee J, Zerr DM, Elward A, et al. Improving surveillance for pediatric Clostridium difficile infection: derivation and validation of an accurate case-finding tool. Pediatr Infect Dis J. 2011;30(3):e38-40.

9. Schmiedeskamp M, Harpe S, Polk R, Oinonen M, Pakyz A. Use of international classification of diseases, ninth revision, clinical modification codes and medication use data to identify nosocomial Clostridium difficile infection. Infect Control Hosp Epidemiol. 2009;30(11):1070-6.

10. Feudtner C, Christakis DA, Connell FA. Pediatric deaths attributable to complex chronic conditions: a population-based study of Washington State, 1980-1997. Pediatrics. 2000;106(1 Pt 2):205-9.

11. McDonald LC, Coignard B, Dubberke E, et al. Recommendations for surveillance of Clostridium 
difficile-associated disease. Infect Control Hosp Epidemiol. 2007;28(2):140-5.

12. Tschudin-Sutter S, Tamma PD, Milstone AM, Perl TM. Predictors of first recurrence of Clostridium difficile infections in children. Pediatr Infect Dis J. 2014;33(4):414-6. doi:10.1097/INF.0000000000000 108.

13. Llor C, Bjerrum L. Antimicrobial resistance: risk associated with antibiotic overuse and initiatives to reduce the problem. Therap Adv Drug Saf. 2014;5(6):229-41.

14. Braykov NP, Morgan DJ, Schweizer ML, et al. Assessment of empirical antibiotic therapy optimisation in six hospitals: an observational cohort study. Lancet Infect Dis. 2014;14(12):1220-7.
15. Hersh AL, Shapiro DJ, Pavia AT, Shah SS. Antibiotic prescribing in ambulatory pediatrics in the United States. Pediatrics. 2011;128(6):1053-61. doi:10. 1542/peds.2011-1337

16. Sharma A, Lokeshwar N. Febrile neutropenia in haematological malignancies. J Postgrad Med. 2005;51(Suppl 1):S42-8.

17. Fisher BT, Sammons JS, Li Y, et al. Variation in risk of hospital-onset Clostridium difficile infection across $\beta$-lactam antibiotics in children with new-onset acute lymphoblastic leukemia. J Pediatr Infect Dis Soc. 2014;3(4):329-35.

18. Sammons JS, Toltzis P. Pitfalls in diagnosis of pediatric Clostridium difficile infection. Infect Dis Clin North Am. 2015;29(3):465-76. 